\title{
On fluid flows in precessing spheres in the mantle frame of reference
}

\author{
Keke Zhang, ${ }^{1, a)}$ Kit H. Chan, ${ }^{2}$ and Xinhao Liao ${ }^{3}$ \\ ${ }_{1}^{1}$ Department of Mathematical Sciences, University of Exeter, EX4 4QE, United Kingdom \\ ${ }^{2}$ Department of Mathematics, The University of Hong Kong, Pokfulam, Hong Kong \\ ${ }^{3}$ Shanghai Astronomical Observatory, Chinese Academy of Sciences, Shanghai 200030, China
}

(Received 20 February 2010; accepted 18 October 2010; published online 16 November 2010)

\begin{abstract}
We investigate, through both asymptotic and numerical analysis, precessionally driven flows of a homogeneous fluid confined in a spherical container that rotates rapidly with angular velocity $\boldsymbol{\Omega}$ and precesses slowly with angular velocity $\boldsymbol{\Omega}_{p}$ about an axis that is fixed in space. The precessionally driven flows are primarily characterized by two dimensionless parameters: the Ekman number $E$ providing the measure of relative importance between the viscous force and the Coriolis force, and the Poincaré number Po quantifying the strength of the Poincaré forcing. When $E$ is small but fixed and $|P o|$ is sufficiently small, we derive a time-dependent asymptotic solution for the weakly precessing flow that satisfies the nonslip boundary condition in the mantle frame of reference. No prior assumption about the spatial-temporal structure of the precessing flow is made in the asymptotic analysis. A solvability condition is derived to determine the spatial structure of the precessing flow, via a selection from a complete spectrum of spherical inertial modes in the mantle frame. The weakly precessing flow within the bulk of the fluid is characterized by an inertial wave moving retrogradely. Direct numerical simulation of the same problem in the same frame of reference shows a satisfactory agreement between the time-dependent asymptotic solution and the nonlinear numerical simulation for sufficiently small Poincaré numbers. (C) 2010 American Institute of Physics. [doi:10.1063/1.3515344]
\end{abstract}

\section{INTRODUCTION}

As an alternative cause for generating and maintaining the geomagnetic field, ${ }^{1,2}$ precessionally driven flows in spherical/spheroidal geometry have been studied for a long time. Energetically, it has been estimated that there is abundant precessional energy to drive the geodynamo. ${ }^{3}$ Experimentally, it has been demonstrated that spatially complex flows, which are usually required for dynamo action, can occur in strongly precessing systems. ${ }^{2,4-7}$ Computationally, it has been convincingly shown that procession-driven flows can indeed generate and sustain magnetic fields. ${ }^{8-10}$ Two special features of the precession problem - the abundant energy and the persistent nature-make it significant in many geophysical and astrophysical problems, providing a strong impetus to the research on the subject. The present paper is concerned with a viscous, incompressible fluid occupying a full sphere that rotates rapidly with angular velocity $\boldsymbol{\Omega}$ and precesses slowly with angular velocity $\boldsymbol{\Omega}_{p}$. The precession vector $\boldsymbol{\Omega}_{p}$ is fixed in space and inclined at an angle $\alpha$ with respect to the rotation vector $\boldsymbol{\Omega}$, and fluid motion is driven by the precessing spherical container only through the viscous coupling between the rigid container and the weakly viscous fluid.

Over the past several decades, the problem of precessing flows in fluid spheres has been investigated by theoretical methods ${ }^{6,11-14}$ and by numerical simulations. ${ }^{5,15-17}$ In previous theoretical studies, a frame of reference rotating about the precession axis $\boldsymbol{\Omega}_{p}$ (the precession frame) is usually

\footnotetext{
${ }^{a)}$ Electronic mail: kzhang@ex.ac.uk.
}

adopted and, furthermore, the precessing flow $\mathbf{u}$ is assumed to be stationary, and is then governed by

$$
\begin{aligned}
& \mathbf{u} \cdot \nabla \mathbf{u}+2 \boldsymbol{\Omega}_{p} \times \mathbf{u}=-\frac{1}{\rho} \nabla p+\nu \nabla^{2} \mathbf{u}, \\
& \nabla \cdot \mathbf{u}=0
\end{aligned}
$$

where $\nu$ is the kinematic viscosity of the fluid, $\rho$ is the fluid density, $p$ is a reduced pressure, and $\mathbf{u}$ is the threedimensional velocity field. It should be noted that, in this particular frame, both the rotation vectors, $\boldsymbol{\Omega}$ and $\boldsymbol{\Omega}_{p}$, are independent of time and that the nonslip boundary condition requires

$$
\mathbf{u}=\mathbf{\Omega} \times \mathbf{r}
$$

on the bounding surface $\mathcal{S}$ of the spherical container, where $\mathbf{r}$ is the position vector. The mathematical analysis in the precession frame becomes particularly convenient by further postulating that the precessing flow is not only stationary but also in the form of rigid-body rotation given by

$$
\mathbf{u}=\boldsymbol{\omega}_{F} \times \mathbf{r},
$$

where $\boldsymbol{\omega}_{F}$ is a constant vector. The remaining task of the analysis is then to determine the stationary vector $\boldsymbol{\omega}_{F}$. By taking into account the viscous effect in the thin viscous boundary layer, Roberts and Stewartson ${ }^{12}$ determined both the amplitude and orientation of the rigid-body rotation. Their analysis was later extended by Busse ${ }^{13}$ who incorporated the weakly nonlinear effect within the boundary layer and established the profile of the relevant differential rotation. Taking advantage of the rigid-body-rotation assump- 
tion, a relatively simple torque-balance approach was also successfully employed to evaluate $\boldsymbol{\omega}_{F}$ by Noir et al.; ${ }^{6}$ see also Ref. 14.

The present investigation attempts to improve the understanding of the nature of the precessionally driven flows in a full sphere through a different asymptotic approach. In our asymptotic analysis, the mantle frame of reference will be adopted and, more significantly, no prior assumptions about the spatial-temporal structure of the precessing flow will be made. For several reasons it is desirable to adopt the mantle frame of reference in the theoretical analysis although it is mathematically more complicated. All the theoretical studies of rotating convection and planetary dynamos are conducted using the mantle frame of reference. In many respects, the mathematical problem of precessionally driven flows in a sphere is similar to that of buoyancy-driven convection and, indeed, Vanyo et al. ${ }^{4}$ in an experimental study demonstrated that the basic elements of precessing flows are largely the same as those in buoyancy-driven convection. Treating the precessing flow in the same frame as that for the convection/ dynamo problem would help develop a unified mathematical theory. Finally, the adoption of the mantle frame is also of practical importance because most geophysical observations/ phenomena such as the core flow are made/described in the Earth's mantle frame and many measurements of the precessing flow in experimental studies are made in the mantle frame.

As discussed by Tilgner, ${ }^{18}$ the analytical work on precessing flows is generally easier in the precession frame; theoretical studies using the mantle frame are much harder. With the time dependence introduced by adopting the mantle frame, it seems inappropriate because of the complicated time-dependent term $\mathbf{r} \times\left[\boldsymbol{\Omega}_{p}(t) \times \boldsymbol{\Omega}\right]$ in the governing equation [see Eqs. (5) and (9) for detail], to make a prior assumption that the precessing flow in the mantle frame is in the form of rigid-body-rotation. This complication may explain why no theoretical studies in the mantle frame of reference, despite its practical importance, have been carried out. In the meantime, it is nontrivial to derive an analytical solution for the precessing flow $\mathbf{u}$ in the mantle frame that satisfies the nonslip condition by transforming the existing rigid-bodyrotation solution together with the stationary boundary-layer solution to the mantle frame. In fact, there does not exist an analytical solution in the mantle frame for the precessing flow $\mathbf{u}$ - the time-dependent mainstream flow plus the timedependent boundary layer solution-in the literature.

As a consequence of using the mantle frame, our theoretical approach has to be both physically and mathematically different from previous ones. Physically, the leadingorder solution in the limits $\nu=0$ and $\boldsymbol{\Omega}_{p}=0$ is described by the complete spectrum of spherical inertial oscillation and waves ${ }^{19,20}$ whose explicit analytical solutions are available. ${ }^{21}$ When the container precesses slowly $\left(\boldsymbol{\Omega}_{p} \neq 0\right)$ and the fluid is slightly viscous $(\nu \neq 0)$, the resulting Poincaré force, through the viscous coupling between the spherical container and the fluid, selects and excites either a single inertial mode or a number of inertial modes from the complete spectrum. Mathematically, in the mantle frame of reference, the selection/excitation process forms a perturbation problem in that both the structure and amplitude of precessionally driven flows are determined without the need to make any prior assumption.

In what follows we shall begin by presenting the governing mathematical equations of the problem in Sec. II. The asymptotic analysis for weakly precessing flows, together with its validation by direct numerical simulation, is discussed in Sec. III. A summary and some remarks are given in Sec. IV.

\section{MATHEMATICAL FORMULATION OF THE PROBLEM}

Consider a viscous, homogeneous fluid confined in a spherical container of radius $r_{o}$. Suppose that the container rotates rapidly with angular velocity $\hat{\mathbf{z}} \Omega$ where $\hat{\mathbf{z}}$ is a unit vector and, at the same time, precesses slowly with angular velocity $\boldsymbol{\Omega}_{p}$ that is fixed in space and inclined at an angle $\alpha$ $(0<\alpha<\pi / 2)$ to $\hat{\mathbf{z}}$. In the numerical analysis validating our asymptotic analytical solution, we shall focus on the case $\alpha=23.5^{\circ}$ which represents the Earth's precession caused by the luni-solar torque. In a frame of reference attached to the spherical container, the mantle frame, processionally driven flows in an incompressible fluid are governed by the dimensional equations ${ }^{17,19}$

$$
\begin{aligned}
\frac{\partial \mathbf{u}}{\partial t} & +\mathbf{u} \cdot \nabla \mathbf{u}+2\left(\Omega \hat{\mathbf{z}}+\boldsymbol{\Omega}_{p}\right) \times \mathbf{u} \\
& =-\frac{1}{\rho} \nabla p+\nu \nabla^{2} \mathbf{u}+\mathbf{r} \times\left[\boldsymbol{\Omega}_{p} \times(\Omega \hat{\mathbf{z}})\right], \\
\nabla \cdot \mathbf{u} & =0,
\end{aligned}
$$

where $\boldsymbol{\Omega}_{p}$ is time-dependent in the mantle frame, $p$ is a reduced pressure, $\mathbf{u}$ is the three-dimensional velocity field $\mathbf{u}$ $=\left(u_{r}, u_{\theta}, u_{\phi}\right)$ with corresponding unit vectors $(\hat{\mathbf{r}}, \hat{\boldsymbol{\theta}}, \hat{\boldsymbol{\phi}})$ in spherical polar coordinates $(r, \theta, \phi)$ with $\theta=0$ at the axis of $\hat{\mathbf{z}} \Omega$ and $r=0$ at the center of the sphere. The final term on the right-hand side of Eq. (5) is known as the Poincaré force, which drives precessional flows against viscous dissipation.

We employ the radius of the sphere, $r_{o}$, as the length scale, $\Omega^{-1}$ as the unit of time, and $\rho r_{o}^{2} \Omega^{2}$ as the unit of pressure, which gives rise to the dimensionless equations

$$
\begin{aligned}
\frac{\partial \mathbf{u}}{\partial t} & +\mathbf{u} \cdot \nabla \mathbf{u}+2\left(\hat{\mathbf{z}}+P o \hat{\boldsymbol{\Omega}}_{p}\right) \times \mathbf{u}+\nabla p \\
& =E \nabla^{2} \mathbf{u}+P o \mathbf{r} \times\left(\hat{\boldsymbol{\Omega}}_{p} \times \hat{\mathbf{z}}\right), \\
\nabla \cdot \mathbf{u} & =0,
\end{aligned}
$$

where the Ekman number, $E=\nu / \Omega r_{o}^{2}$, provides the measure of relative importance between the typical viscous force and the Coriolis force, and the Poincaré number, $P o= \pm\left|\boldsymbol{\Omega}_{p}\right| / \Omega$, quantifies the strength of the precessional forcing. Positive $P o$ corresponds to the case of prograde precession while $P o<0$ indicates retrograde precession. In the mantle frame, the dimensionless precession vector $\hat{\boldsymbol{\Omega}}_{p}$ is given by 


$$
\begin{aligned}
\hat{\boldsymbol{\Omega}}_{p}= & \sin \alpha[\hat{\mathbf{r}} \sin \theta \cos (\phi+t)+\hat{\boldsymbol{\theta}} \cos \theta \cos (\phi+t) \\
& -\hat{\boldsymbol{\phi}} \sin (\phi+t)]+\hat{\mathbf{z}} \cos \alpha .
\end{aligned}
$$

The precessing flow on the bounding surface $\mathcal{S}$ of the spherical container is at rest, which requires

$$
\hat{\mathbf{r}} \cdot \mathbf{u}=\hat{\mathbf{r}} \times \mathbf{u}=0 .
$$

The problem defined by Eqs. (7) and (8) subject to the boundary conditions (10) will be solved asymptotically for an arbitrarily small but fixed $E$ with sufficiently small $P o$.

\section{ASYMPTOTIC ANALYSIS IN THE MANTLE FRAME}

In all that follows, $\mathbf{u}$ and $p$ are understood to mean the flow velocity and pressure seen in the mantle frame of reference. For weakly precessing flows, we may neglect the nonlinear term $\mathbf{u} \cdot \nabla \mathbf{u}$ in Eq. (7) (see further discussion regarding the nonlinear effect at the end of this section) by considering the linearized equations

$$
\frac{\partial \mathbf{u}}{\partial t}+2 \hat{\mathbf{z}} \times \mathbf{u}+\nabla p=E \nabla^{2} \mathbf{u}+P o\left[\mathbf{r} \times\left(\hat{\boldsymbol{\Omega}}_{p} \times \hat{\mathbf{z}}\right)-2 \hat{\boldsymbol{\Omega}}_{p} \times \mathbf{u}\right],
$$

$\nabla \cdot \mathbf{u}=0$.

Our asymptotic analysis will not make any prior assumption about the structure of the flow. It will be based on the following physical and mathematical observations. First, the analytical solution to Eqs. (11) and (12) in the limit $P o=0$ and $E=0$ is available, ${ }^{21}$ which provides the necessary framework for constructing the leading-order solution to the interior precessing flow in the mantle frame. Naturally, an asymptotic problem describing weakly precessing flows for $|P o| \ll 1$ and $E \ll 1$ may be regarded as a small perturbation to the leading-order solution at $P o=0$ and $E=0$, forming a mathematically tractable asymptotic problem. Second, the Poincaré forcing, $\operatorname{Por} \times\left(\hat{\boldsymbol{\Omega}}_{p} \times \hat{\mathbf{z}}\right)$, is diurnal and equatorially antisymmetric in the mantle frame. Consequently, only the spherical inertial modes with the same symmetry are required in the asymptotic analysis. Finally, if we treat the Poincaré forcing in Eq. (11) the similar way as we treat the buoyancy force in convection, the asymptotic method used in convection by Zhang and $\mathrm{Liao}^{22}$ may be modified to solve the precession problem.

Those observations lead to an asymptotic expansion of the spherical precessing flow $\mathbf{u}$ for $|P o| \ll 1$ and $E \ll 1$ in the mantle frame

$$
\mathbf{u}=\sum_{m n k} \mathcal{A}_{m n k}\left\{\left(\mathbf{u}_{m n k}+\hat{\mathbf{u}}_{m n k}\right)+\left[\widetilde{\mathbf{u}}_{m n k}+\left(\hat{\mathbf{r}} \cdot \hat{\mathbf{u}}_{m n k}\right) \hat{\mathbf{r}}\right]\right\} e^{\mathrm{i} t},
$$

where $\mathrm{i}=\sqrt{-1}, \mathcal{A}_{\text {mnk }}$ are complex coefficients to be determined, and the triple index $-m$ is the azimuthal wavenumber and $n$ and $k$ represent roughly the axial and radial wavenumbers, respectively-is adopted to specify a spherical inertial mode $\mathbf{u}_{m k n}$ that satisfies

$$
\begin{aligned}
& \mathrm{i} 2 \sigma_{m n k} \mathbf{u}_{m n k}+2 \hat{\mathbf{z}} \times \mathbf{u}_{m n k}+\nabla p_{m n k}=0, \\
& \nabla \cdot \mathbf{u}_{m n k}=0
\end{aligned}
$$

where $\sigma_{m n k}$ is the half-frequency of the mode with $\left|\sigma_{m n k}\right|$ $<1$, subject to the inviscid boundary condition $\hat{\mathbf{r}} \cdot \mathbf{u}_{m n k}=0$ on $\mathcal{S}$. In the asymptotic expansion (13), viscous action on each inertial mode $\mathbf{u}_{m n k}$ induces a thin viscous boundary layer on $\mathcal{S}$ with major tangential component $\widetilde{\mathbf{u}}_{m n k}$ (i.e., $\hat{\mathbf{r}} \cdot \widetilde{\mathbf{u}}_{m n k}=0$ ) and small normal component $\hat{\mathbf{n}} \cdot \hat{\tilde{\mathbf{u}}}_{m n k}$. By producing a normal mass flux from, or sucking the interior fluid into, the thin boundary layer, the viscous effect drives the secondary interior flow $\hat{\mathbf{u}}_{m n k}$ and communicates to the interior fluid. Here a precessing flow (13) u for $\mid P o \ll 1$ and $E \ll 1$ is similar to that of convective flow $^{23}$ in which the velocity is decomposed into viscous boundary layers and interior inviscid inertial waves. The pressure $p$ can be expanded in a similar way. It should be pointed out that the asymptotic expansion (13) may be used to represent the spatial structure of any physically acceptable flow satisfying Eq. (12) in rapidly rotating fluid spheres. In other words, no prior assumptions about the spatial-temporal structure of the solution are made in the asymptotic analysis.

The spatial symmetry of the Poincare force, $\mathbf{r} \times\left(\hat{\boldsymbol{\Omega}}_{p}\right.$ $\times \hat{\mathbf{z}}$ ), implies that the asymptotic expansion (13) needs only the spherical inertial modes, $\left(\mathbf{p}_{m n k}, \mathbf{u}_{m n k}\right)$, obeying

$$
\begin{aligned}
& \left(p_{m n k}, \hat{\mathbf{r}} \cdot \mathbf{u}_{m n k}, \hat{\boldsymbol{\theta}} \cdot \mathbf{u}_{m n k}, \hat{\boldsymbol{\phi}} \cdot \mathbf{u}_{m n k}\right)(r, \theta, \phi) \\
& \quad=\left(-p_{m n k},-\hat{\mathbf{r}} \cdot \mathbf{u}_{m n k}, \hat{\boldsymbol{\theta}} \cdot \mathbf{u}_{m n k},-\hat{\boldsymbol{\phi}} \cdot \mathbf{u}_{m n k}\right)(r, \pi-\theta, \phi) .
\end{aligned}
$$

It follows that the explicit analytical expressions for $\mathbf{u}_{m n k}$ and $p_{m n k}$ required in Eq. (13) are given by

$$
\begin{aligned}
& p_{m n k}=\sum_{i=0}^{k} \sum_{j=0}^{k-i} \mathcal{C}_{m k i j} \sigma^{2 i}\left(1-\sigma^{2}\right)^{j} r^{m+2(i+j)+1} \\
& \quad \times \sin ^{2 j+m} \theta \cos ^{2 i+1} \theta e^{\mathrm{i} m \phi}, \\
& \hat{\mathbf{r}} \cdot \mathbf{u}_{m n k}=-\frac{i}{2} \sum_{i=0}^{k} \sum_{j=0}^{k-i} \mathcal{C}_{m k i j} r^{m+2(i+j)} \\
& \\
& \times\left[\sigma_{m n k}^{2}(m+2 j)+m \sigma_{m n k}-(2 i+1)\left(1-\sigma_{m n k}^{2}\right)\right] \\
& \times \sigma_{m n k}^{2 i-1}\left(1-\sigma_{m n k}^{2}\right)^{j-1} \sin ^{m+2 j} \theta \cos ^{2 i+1} \theta e^{\mathrm{i} m \phi},
\end{aligned}
$$

$$
\begin{aligned}
\hat{\boldsymbol{\theta}} \cdot \mathbf{u}_{m n k}= & -\frac{\mathrm{i}}{2} \sum_{i=0}^{k} \sum_{j=0}^{k-i} \mathcal{C}_{m k i j} r^{m+2(i+j)}\left\{\left[\sigma_{m n k}^{2}(m+2 j)+m \sigma_{m n k}\right]\right. \\
& \left.\times \cos ^{2} \theta+(2 i+1)\left(1-\sigma_{m n k}^{2}\right) \sin ^{2} \theta\right\} \\
& \times \sigma_{m n k}^{2 i-1}\left(1-\sigma_{m n k}^{2}\right)^{j-1} \sin ^{m+2 j-1} \theta \cos ^{2 i} \theta e^{\mathrm{i} m \phi},
\end{aligned}
$$




$$
\begin{aligned}
\hat{\boldsymbol{\phi}} \cdot \mathbf{u}_{m n k}= & \frac{1}{2} \sum_{i=0}^{k} \sum_{j=0}^{k-i} \mathcal{C}_{m k i j} r^{m+2(i+j)} \sigma_{m n k}^{2 i}\left[(m+2 j)+m \sigma_{m n k}\right] \\
& \times\left(1-\sigma_{m n k}^{2}\right)^{j-1} \sin ^{m+2 j-1} \theta \cos ^{2 i+1} \theta e^{\mathrm{i} m \phi},
\end{aligned}
$$

where $m \geq 0, k=0,1,2, \ldots$, and $n=1,2, \ldots,(2 k+1), \mathcal{C}_{m k i j}$ is defined as

$$
\mathcal{C}_{m k i j}=\frac{(-1)^{i+j}[2(m+k+i+j)+1] ! !}{2^{j+1}(2 i+1) ! !(k-i-j) ! i ! j !(m+j) !}
$$

while the half frequencies, $\sigma_{m n k}$, are solutions of

$$
\begin{aligned}
0= & \sum_{j=0}^{k} \frac{(-1)^{j}[2(2 k+m-j+1)] !}{j ![2(k-j)+1] !(2 k+m-j+1) !} \sigma_{m n k}^{2(k-j)} \\
& \times\left[(2 k-2 j+m+1) \sigma_{m n k}-(2 k-2 j+1)\right] .
\end{aligned}
$$

Here, for each given $k \geq 0$, the $2 k+1$ distinct real solutions of Eq. (20) can be arranged according to their size

$$
0<\left|\sigma_{m 1 k}\right|<\left|\sigma_{m 2 k}\right|<\left|\sigma_{m 3 k}\right|, \ldots,<\left|\sigma_{m n k}\right|<\ldots
$$

with $\sigma_{m n k}$ representing the $n$th smallest absolute solution of Eq. (20) and $1 \leq n \leq(2 k+1)$. For example, Eq. (20) yields $\sigma_{110}=1 / 2$ when $m=1, n=1$, and $k=0$, corresponding to the simplest inertial mode $\left[p_{110}, \mathbf{u}_{110}\right]$.

Consider first the solution for the thin viscous boundary layer on $\mathcal{S}$. On substitution of Eq. (13) into Eqs. (11) and (12), the leading-order solution of the viscous boundary layer is described by the equations

$\sum_{m n k} \mathcal{A}_{m n k}\left[i \widetilde{\mathbf{u}}_{m n k}+2 \hat{\mathbf{z}} \times \widetilde{\mathbf{u}}_{m n k}-\hat{\mathbf{r}} E^{-1 / 2}\left(\frac{\partial \widetilde{p}_{m n k}}{\partial \xi}\right)-\frac{\partial^{2} \widetilde{\mathbf{u}}_{m n k}}{\partial \xi^{2}}\right]=0$, $\frac{\partial}{\partial \xi}\left(\sum_{m n k} \mathcal{A}_{m n k} \hat{\mathbf{r}} \cdot \hat{\tilde{\mathbf{u}}}_{m n k}\right)=E^{1 / 2} \hat{\mathbf{r}} \cdot \nabla\left[\hat{\mathbf{r}} \times\left(\sum_{m n k} \mathcal{A}_{m n k} \widetilde{\mathbf{u}}_{m n k}\right)\right]$,

where we have introduced a stretched boundary-layer variable $\xi$ for which $\hat{\mathbf{r}} \cdot \nabla=-E^{-1 / 2} \partial / \partial \xi$ with $\xi=0$ at $\mathcal{S}$ while $\xi=\infty$ defines the outer edge of the thin boundary layer. It is mathematically convenient to derive a fourth-order differential equation by applying the operators $\hat{\mathbf{r}} \times$ and $\hat{\mathbf{r}} \times \hat{\mathbf{r}} \times$ to Eq. (21), and then combining the two resulting equations into a single equation

$$
\left[\left(\frac{\partial^{2}}{\partial \xi^{2}}-\mathrm{i}\right)^{2}+4(\hat{\mathbf{z}} \cdot \hat{\mathbf{r}})^{2}\right]\left(\sum_{m n k} \mathcal{A}_{m n k} \widetilde{\mathbf{u}}_{m n k}\right)=0
$$

which is solved subject to the four boundary conditions

$$
\begin{aligned}
& \left(\sum_{m n k} \mathcal{A}_{m n k} \widetilde{\mathbf{u}}_{m n k}\right)_{\xi=0}=-\left(\sum_{m n k} \mathcal{A}_{m n k} \mathbf{u}_{m n k}\right)_{r=1} \\
& \left(\sum_{m n k} \mathcal{A}_{m n k} \frac{\partial^{2} \widetilde{\mathbf{u}}_{m n k}}{\partial \xi^{2}}\right)_{\xi=0}=-\sum_{m n k} \mathcal{A}_{m n k}\left[i \mathbf{u}_{m n k}+2(\hat{\mathbf{z}} \cdot \hat{\mathbf{r}}) \hat{\mathbf{r}} \mathbf{u}_{m n k}\right]_{r=1},
\end{aligned}
$$

$$
\begin{aligned}
& \left(\sum_{m n k} \mathcal{A}_{m n k} \widetilde{\mathbf{u}}_{m n k}\right)_{\xi=\infty}=0, \\
& \left(\sum_{m n k} \mathcal{A}_{m n k} \frac{\partial^{2} \widetilde{\mathbf{u}}_{m n k}}{\partial \xi^{2}}\right)_{\xi=\infty}=0 .
\end{aligned}
$$

Here the explicit expression for $\mathbf{u}_{m n k}$ is given by Eqs. (17)-(19). The fourth-order Eq. (23), together with the four boundary conditions (24)-(27), can be solved to give a boundary-layer solution in which the coefficients $\mathcal{A}_{m n k}$ remain undetermined.

Consider now the interior solution whose asymptotic match to the boundary-layer solution would determine the values of $\mathcal{A}_{m n k}$. Substituting Eq. (13) into Eq. (11), multiplying the resulting equation by $\mathbf{u}_{m n k}^{*}$, the complex conjugate of $\mathbf{u}_{m n k}$, making use of mass conservation (12) and then integrating over the sphere, we obtain the solvability condition for the interior problem

$$
\begin{aligned}
\mathrm{i}(1 & \left.-2 \sigma_{m n k}\right) \mathcal{A}_{m n k} \int_{0}^{2 \pi} \int_{0}^{\pi} \int_{0}^{1}\left|\mathbf{u}_{m n k}\right|^{2} r^{2} \sin \theta r^{2} d r d \theta d \phi+E^{1 / 2} \sum_{m^{\prime} n^{\prime} k^{\prime}} \mathcal{A}_{m^{\prime} n^{\prime} k^{\prime}} \int_{0}^{2 \pi} \int_{0}^{\pi}\left(p_{m n k}^{*}\right)_{r=1} \\
\times & {\left[\int_{0}^{\infty} \hat{\mathbf{r}} \cdot \nabla \times\left(\hat{\mathbf{r}} \times \widetilde{\mathbf{u}}_{m^{\prime} n^{\prime} k^{\prime}}\right) d \xi\right] \sin \theta d \theta d \phi } \\
= & P o \int_{0}^{2 \pi} \int_{0}^{\pi} \int_{0}^{1} \mathbf{u}_{m n k}^{*} \cdot\left[\mathbf{r} \times\left(\hat{\mathbf{\Omega}}_{p} \times \hat{\mathbf{z}}\right)\right] r^{2} \sin \theta d r d \theta d \phi-2 P o \sum_{m^{\prime} n^{\prime} k^{\prime}} \mathcal{A}_{m^{\prime} n^{\prime} k^{\prime}} \\
& \times \int_{0}^{2 \pi} \int_{0}^{\pi} \int_{0}^{1} \mathbf{u}_{m n k}^{*} \cdot\left(\hat{\boldsymbol{\Omega}}_{p} \times \mathbf{u}_{m^{\prime} n^{\prime} k^{\prime}}\right) r^{2} \sin \theta d r d \theta d \phi,
\end{aligned}
$$


where $p_{m n k}^{*}$ denotes the complex conjugate of $p_{m n k}$ and the indices, $m, n$, and $k$, take all permissible values. In deriving Eq. (28), we have used the fact ${ }^{21}$ that

$$
\int_{0}^{2 \pi} \int_{0}^{\pi} \int_{0}^{1}\left(\mathbf{u}_{m n k}^{*} \cdot \nabla^{2} \mathbf{u}_{m n k}\right) r^{2} \sin \theta d r d \theta d \phi \equiv 0 .
$$

In the solvability condition (28), we notice, by performing direct integration using the expressions (9) and (17)-(19), that

$$
\int_{0}^{2 \pi} \int_{0}^{\pi} \int_{0}^{1} \mathbf{u}_{m n k}^{*} \cdot\left[\mathbf{r} \times\left(\hat{\boldsymbol{\Omega}}_{p} \times \hat{\mathbf{z}}\right)\right] r^{2} \sin \theta r^{2} d r d \theta d \phi=0
$$

for all indices $m, n$, and $k$ except for $m=1, n=1$, and $k=0$. This implies that the solvability system (28) can be reduced to

$$
\mathcal{A}_{\text {mnk }}=0, \text { except for } m=1, n=1, k=0
$$

with $\mathcal{A}_{110}$ given by the equation

$$
\begin{aligned}
E^{1 / 2} & \mathcal{A}_{110} \int_{0}^{2 \pi} \int_{0}^{\pi}\left[p_{110}^{*}\right]_{r=1} \\
& \times\left[\int_{0}^{\infty} \hat{\mathbf{r}} \cdot \nabla \times\left(\hat{\mathbf{r}} \times \widetilde{\mathbf{u}}_{110}\right) d \xi\right] \sin \theta d \theta d \phi \\
= & P o \int_{0}^{2 \pi} \int_{0}^{\pi} \int_{0}^{1} \mathbf{u}_{110}^{*} \cdot\left[\mathbf{r} \times\left(\hat{\mathbf{\Omega}}_{p} \times \hat{\mathbf{z}}\right)\right] r^{2} \sin \theta d r d \theta d \phi \\
& +\mathrm{i} P o \cos \alpha \mathcal{A}_{110} \int_{0}^{2 \pi} \int_{0}^{\pi} \int_{0}^{1}\left|\mathbf{u}_{110}\right|^{2} r^{2} \sin \theta d r d \theta d \phi .
\end{aligned}
$$

Note that the $O(P o \sin \alpha)$ terms do not make contribution to the primary solution with the azimuthal wavenumber $m=1$ at leading order. We have thus established that there exists only one inertial mode in the mantle frame of reference, $\mathbf{u}_{110}$, that can be excited and maintained by the Poincaré forcing via the viscous coupling.

In Eq. (29), the pressure $p_{110}$ and the velocity $\mathbf{u}_{110}$ can be obtained simply by setting $m=1, n=1$, and $k=0$, together with $\sigma_{110}=1 / 2$, in Eqs. (16)-(19). Though the boundarylayer solution, $\mathcal{A}_{110} \widetilde{\mathbf{u}}_{110}$, can be readily derived by solving Eq. (23) subject to the conditions (24)-(27) for a single inertial mode $\mathbf{u}_{110}$, the boundary-layer mass flux in Eq. (29) needs careful manipulation. It can be shown that the mass flux takes the form

$$
\begin{aligned}
& {\left[\mathcal{A}_{110} \int_{0}^{\infty} \hat{\mathbf{r}} \cdot \nabla \times\left(\hat{\mathbf{r}} \times \widetilde{\mathbf{u}}_{110}\right) d \xi\right]} \\
& \quad=\mathcal{A}_{110} \times \frac{3 \sqrt{2}}{8}\left[\frac{\partial}{\partial x}\left(T_{1}(x) \sqrt{1-x^{2}}\right)+\frac{\mathrm{i} T_{2}(x)}{\sqrt{1-x^{2}}}\right] e^{\mathrm{i} \phi},
\end{aligned}
$$

where $x=\cos \theta$ and

$$
\begin{aligned}
& T_{1}(x)=\frac{\mathrm{i} x}{\sqrt{|1+2 x|}}+\frac{x(1+2 x)}{\sqrt{|1+2 x|^{3}}}+\frac{\mathrm{i} x}{\sqrt{|1-2 x|}}+\frac{x(1-2 x)}{\sqrt{|1-2 x|^{3}}}, \\
& T_{2}(x)=\frac{-x}{\sqrt{|1+2 x|}}+\frac{\mathrm{i} x(1+2 x)}{\sqrt{|1+2 x|^{3}}}+\frac{x}{\sqrt{|1-2 x|}}-\frac{\mathrm{i} x(1-2 x)}{\sqrt{|1-2 x|^{3}}} .
\end{aligned}
$$

We are now in a position to determine, by carrying out the relevant integrations, each term in the solvability condition (29)

$$
\begin{aligned}
& E^{1 / 2} \int_{0}^{2 \pi} \int_{0}^{\pi}\left[p_{110}^{*}\right]_{r=1}\left[\mathcal{A}_{110} \int_{0}^{\infty} \hat{\mathbf{r}} \cdot \nabla \times\left(\hat{\mathbf{r}} \times \widetilde{\mathbf{u}}_{110}\right) d \xi\right] \\
& \quad \times \sin \theta d \theta d \phi \\
& \quad=\frac{E^{1 / 2} \mathcal{A}_{110} 9 \pi \sqrt{2}}{70}[(19+9 \sqrt{3})-\mathrm{i}(19-9 \sqrt{3})], \\
& P o \int_{0}^{2 \pi} \int_{0}^{\pi} \int_{0}^{1} \mathbf{u}_{110}^{*} \cdot\left[\mathbf{r} \times\left(\hat{\mathbf{\Omega}}_{p} \times \hat{\mathbf{z}}\right)\right] \times r^{2} \sin \theta d r d \theta d \phi \\
& \quad=\frac{\mathrm{i} P o \sin \alpha 8 \pi}{5}, \\
& \mathrm{i} \text { Po } \cos \alpha \mathcal{A}_{110} \int_{0}^{2 \pi} \int_{0}^{\pi} \int_{0}^{1}\left|\mathbf{u}_{110}\right|^{2} r^{2} \sin \theta d r d \theta d \phi, \\
& \quad=\frac{\mathrm{i} P o \cos \alpha \mathcal{A}_{110} 12 \pi}{5} .
\end{aligned}
$$

With the above results, $\mathcal{A}_{110}$ can be readily determined from Eq. (29). Substitution of all $\mathcal{A}_{m n k}$, as well as $\widetilde{\mathbf{u}}_{110}$ and $\mathbf{u}_{110}$, into the asymptotic expansion (13) yields the leading-order time-dependent asymptotic solution in the mantle frame of reference, describing the weakly precessing flows satisfying the nonslip boundary condition

$$
\begin{aligned}
\mathbf{u}= & \frac{\mathrm{i} P o \sin \alpha\left[\mathrm{i}\left(\beta^{-} E^{1 / 2}+P o \cos \alpha\right)+\beta^{+} E^{1 / 2}\right]}{2\left[\left(\beta^{-} E^{1 / 2}+P o \cos \alpha\right)^{2}+\left(\beta^{+} E^{1 / 2}\right)^{2}\right]} \\
& \times\left[2 r(\hat{\boldsymbol{\phi}} \cos \theta-\mathrm{i} \hat{\boldsymbol{\theta}})+(1-\cos \theta)(\mathrm{i} \hat{\boldsymbol{\theta}}+\hat{\boldsymbol{\phi}}) e^{\gamma^{+} E^{-1 / 2}(1-r)}\right. \\
& \left.+(1+\cos \theta)(\mathrm{i} \hat{\boldsymbol{\theta}}-\hat{\boldsymbol{\phi}}) e^{\gamma^{-} E^{-1 / 2}(1-r)}\right] e^{\mathrm{i}(\phi+t)},
\end{aligned}
$$

where the real part of the expression is taken and

$$
\begin{aligned}
& \beta^{+}=\frac{3}{56}(19 \sqrt{2}+9 \sqrt{6}), \\
& \beta^{-}=\frac{3}{56}(19 \sqrt{2}-9 \sqrt{6}), \\
& \gamma^{+}=-\frac{\sqrt{2}}{2}\left[1+\frac{\mathrm{i}(1+2 \cos \theta)}{|1+2 \cos \theta|}\right]|1+2 \cos \theta|^{1 / 2}, \\
& \gamma^{-}=-\frac{\sqrt{2}}{2}\left[1+\frac{\mathrm{i}(1-2 \cos \theta)}{|1-2 \cos \theta|}\right]|1-2 \cos \theta|^{1 / 2} .
\end{aligned}
$$

Evidently, the analytical expression (30) represents a retrogradely traveling wave of period $2 \pi$. Far away from the 


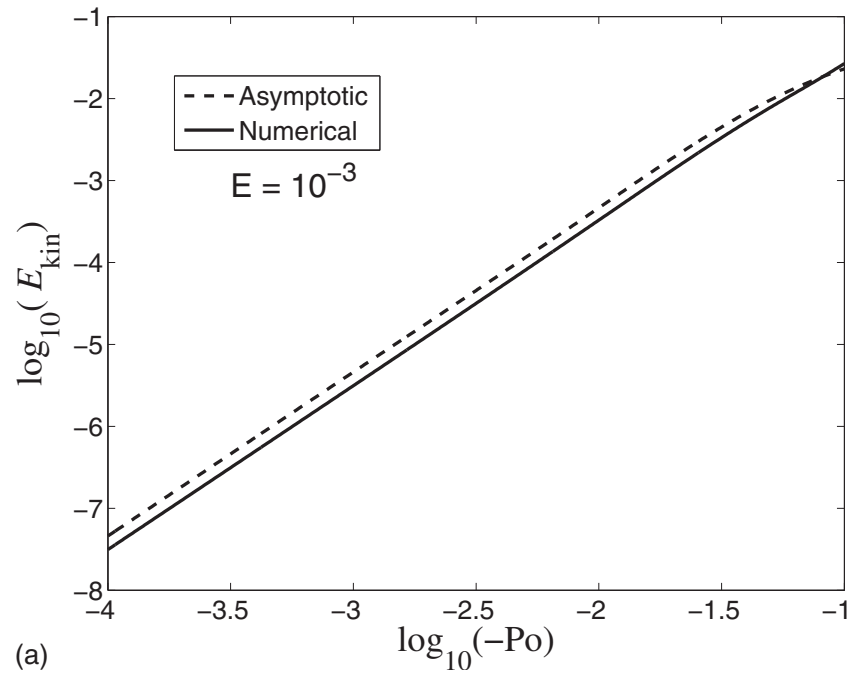

(a)

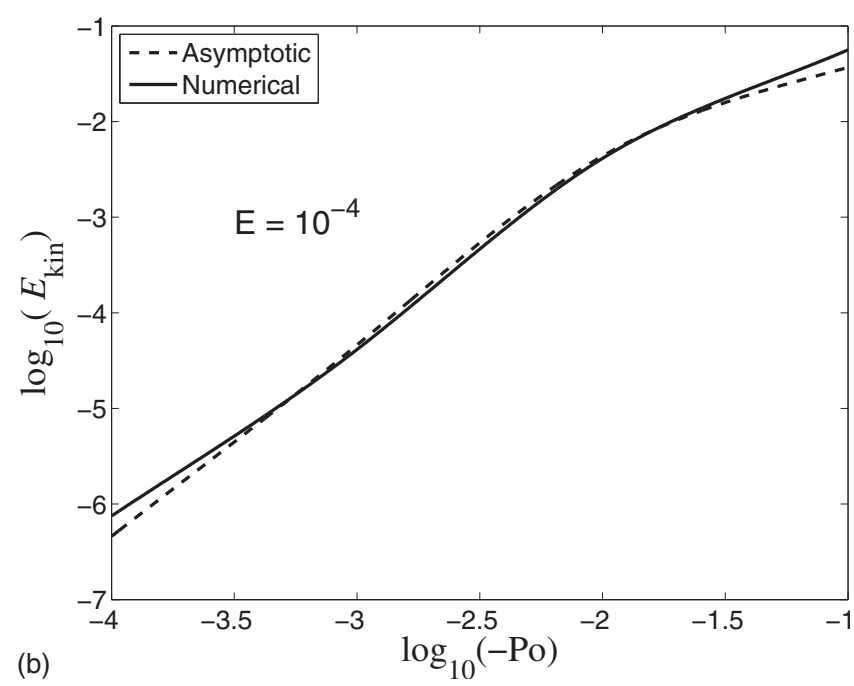

FIG. 1. Kinetic energies, $E_{\text {kin }}$, of the retrogradely precessing flow $(P o<0)$ based on the analytical expression (30) and from the three-dimensional direct numerical simulation, plotted as a function of $P o$ for (a) $E=10^{-3}$ and (b) $E=10^{-4}$ with $\alpha=23.5^{\circ}$.

viscous boundary layer where $\left[E^{-1 / 2}(1-r)\right] \gg 1$, Eq. (30) suggests that the interior flow is in the form of an inertial wave traveling azimuthally and retrogradely. In Eq. (30), there exist two critical colatitudes, $\theta_{c}=\pi / 3$ and $\theta_{c}=2 \pi / 3$, at which the boundary-layer solution breaks down as it thickens to $\mathrm{O}\left(E^{2 / 5}\right)$, as discussed by Roberts and Stewartson. ${ }^{12}$ However, it is generally believed, and has been demonstrated, that the effect of the singularities is weak and insignificant since the mass flux from the critical regions is much smaller than that from the rest of the boundary layer. ${ }^{12,13,15,16}$ Since the nonlinear effect has been neglected in the analysis, the expression (30) is strictly correct if $|P o \sin \alpha| \ll \mathrm{O}\left(E^{1 / 2}\right)$.

The asymptotic solution (30) in the mantle frame, despite its relative simplicity, can be compared directly to fully nonlinear numerical simulations performed at small but fixed $E$ for sufficiently small $P o$ based on a finite element method. ${ }^{24}$ Figure 1 (the dashed lines) shows kinetic energies of the precessing flows per volume, $E_{\text {kin }}$, defined as
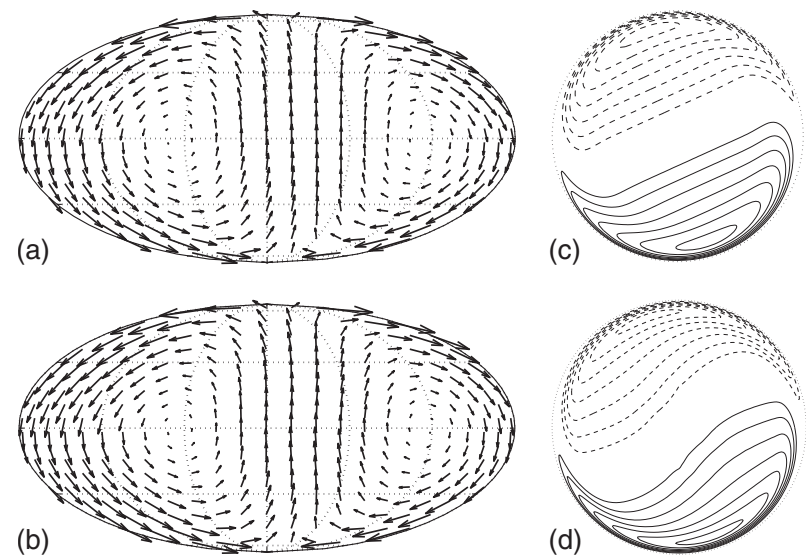

FIG. 2. Flow vectors on the spherical surface at $r=0.85$ (the Mollweide equal area projection) in the drifting frame of the wave: (a) from the asymptotic solution (30) and (b) from the corresponding numerical simulation. Contours of the velocity component $u_{\theta}$ at the equatorial plane in the drifting frame: (c) from the asymptotic solution (30) and (d) from the corresponding numerical simulation. In (c) and (d), dashed contours denote $u_{\theta}<0$ while for solid contours $u_{\theta}>0$. The parameters for both the asymptotic and numerical solutions are $P o=-10^{-4}$ and $E=10^{-3}$ with $\alpha=23.5^{\circ}$.

$$
E_{\mathrm{kin}}=\frac{1}{2}\left(\frac{3}{4 \pi}\right) \int_{0}^{2 \pi} \int_{0}^{\pi} \int_{0}^{1}|\mathbf{u}(r, \theta, \phi)|^{2} r^{2} \sin \theta d r d \theta d \phi,
$$

where the flow $\mathbf{u}$ is given by the expression (30), for two different values of $E$ as a function of Po. Numerical solutions (the solid lines) are also displayed in the same figure for the purpose of comparison. It should be noted that the dependence of the kinetic energies $E_{\text {kin }}$ on $P o$ for a fixed $E$ is, generally speaking, not linear, which is clearly shown in the analytical expression (30) and reflected in Fig. 1(b). In order to illustrate the spatial structure of the precessing flow, the flow vectors in the mantle frame of reference on a whole spherical surface just below the container's envelope at $r=0.85$ are plotted in the drifting frame of the traveling wave in Fig. 2(a), showing the flow structure obtained from Eq. (30) for $P o=-10^{-4}, E=10^{-3}$, and $\alpha=23.5^{\circ}$. Moreover, contours of $u_{\theta}$ for the precessing flow at the equatorial plane are depicted in Fig. 2(c). They should be compared to the corresponding nonlinear numerical solution obtained at exactly the same parameters which are shown in Figs. 2(b) and 2(d).

It should be pointed out that, because a special property of the mainstream flow $\mathbf{u}$-which satisfies $\mathbf{u} \cdot \nabla \mathbf{u}=\nabla \Phi$ far away from the boundary layer, where $\Phi$ is a potential-the nonlinear effect occurs only in the viscous boundary layer at next order. This explains why a reasonable agreement between the expression (30) and fully nonlinear numerical simulations is achieved beyond the limit of the linear analysis.

\section{SUMMARY AND REMARKS}

We have studied, through both asymptotic analysis and numerical simulation, precessionally driven flows confined in a fluid sphere rotating rapidly with angular velocity $\boldsymbol{\Omega}$ that itself precesses slowly about an axis fixed in an inertial frame. The precessionally driven flow in the sphere is mainly 
characterized by two parameters: the Ekman number $E$ and the Poincaré number $P o$. When $E \ll 1$ and $|P o|$ is sufficiently small, the precessing flow is characterized by an inertial wave moving retrogradely. The analytical expression (30) for the weakly precessing flow in the mantle frame is checked with fully nonlinear numerical simulation using the elementby-element (EBE) finite element method. We have focused on the spherical cavity in that the container is only viscously coupled with the fluid. An asymptotic analysis for a spheroidal cavity of arbitrary eccentricity, where both viscous and topographic couplings take place, is much more complicated-because general spheroidal coordinates must be employed in the analysis-and will be carried out in the future.

In comparison to the previous theoretical studies, the mantle frame of reference is adopted for the first time in the asymptotic analysis of spherical precessing flows. The analytical expression (30) represents the first asymptotic solution of the precessing flow in literature that is in the mantle frame and satisfies the nonslip boundary condition. Although the expression (30) may be, in principle, obtainable by transforming the rigid-body-rotation solution ${ }^{6,12,13}$ to the mantle frame, our theoretical approach is different from the previous studies. In the asymptotic expansion (13) and the subsequent analysis, we do not make any prior assumption about the precessing flow. It is the general solvability condition (28) that selects/determines the spatial structure of the timedependent precessing flow in the mantle frame. In this respect, our asymptotic analysis-a selection process via the general solvability condition (28) from a complete spectrum of spherical inertial modes-underpins the nature of precessionally driven flows in a more physical way.

The analytical expression (30) is also geophysically important and practically useful. It is well known that the extremely small value of the Ekman number, a consequence of the Earth's rapid rotation and the small viscosity in the Earth's liquid core, causes severe difficulties in numerical modeling of core dynamics. Even with modern powerful supercomputers, it is still difficult to achieve the geophysically realistic value, which is $\mathrm{O}\left(10^{-9}\right)<E<\mathrm{O}\left(10^{-15}\right)$. Our analytical expression (30) is valid for an arbitrarily small Ekman number and, thus, can be employed to understand some aspects of the Earth's core dynamics for geophysically realistic $E$. A geophysically important question is whether the precessionally driven flow in the Earth's fluid core has a sufficiently large amplitude $\mathrm{O}\left(10^{-4} \mathrm{~m} / \mathrm{s}\right)$. Define the dimensional average speed, $U$, of the precessing flow as

$$
U=\left(\frac{1}{V} \int_{V}|\mathbf{u}|^{2} d V\right)^{1 / 2}\left(r_{o} \Omega\right)
$$

where $\mathbf{u}$ is given by Eq. (30), $V=4 \pi / 3, r_{o}=3.485 \times 10^{6} \mathrm{~m}$, $\Omega=7.27 \times 10^{-5} / \mathrm{s}$. By using Eq. (30), we obtain $U$ at leading order

$$
U=\left[\frac{2(P o \sin \alpha)^{2} / 5}{\left(\beta^{-} E^{1 / 2}+P o \cos \alpha\right)^{2}+\left(\beta^{+} E^{1 / 2}\right)^{2}}\right]^{1 / 2}\left(r_{o} \Omega\right) .
$$

Taking $P o=10^{-7}, \alpha=23.5^{\circ}$, appropriate for the Earth, we find that $U=2.43 \times 10^{-2} \mathrm{~m} / \mathrm{s}$ for $E=10^{-8}$ and $U=7.67$ $\times 10^{-2} \mathrm{~m} / \mathrm{s}$ for $E=10^{-9}$. In other words, the Earth's precession via the viscous coupling can drive geophysically significant flows in its fluid core. Lorenzani and Tilgner ${ }^{25}$ arrived at a similar conclusion but with a smaller amplitude of the flow. The difference may be attributable to a finite ellipticity, which changes the resonance condition of spherical inertial modes, adopted in their estimate. It is also important to point out that, owing to the diurnal period of precessionally driven flows, the geomagnetic variation on this timescale would be screened out by the lower mantle.

The essential theoretical idea used in this study is, although differing in detail, largely similar to that developed for the asymptotic solution of thermal convection in rapidly rotating fluid spheres. ${ }^{26}$ Despite the fact that the mathematical analysis of the precessing flow in the mantle frame of reference is more complicated and lengthy, it offers a highly desirable potentiality that we may unify the mathematical theories of buoyancy-driven convection and precessionally driven flows. This unified approach is particularly significant for constructing a planetary dynamo model in that both driving mechanisms-convection and precession-are believed to be energetically important. An indication from this work is that a unified asymptotic theory for both convection and precession in the same frame of reference is within our reach.

\section{ACKNOWLEDGMENTS}

K.Z. expresses his thanks to Professor F. H. Busse, Professor P. H. Roberts, and Professor A. Tilgner for helpful discussions about the problem. K.Z. is supported by UK STFC/NERC grants, K.H.C. by Hong Kong RGC grant (Grant No. 700308), and X.L. by NSFC (Grant No. 10633030), STCSM (Grant No. 08XD14052), and CAS grants. The numerical computation is supported by Shanghai Supercomputer Center.

${ }^{1}$ E. C. Bullard, "The magnetic flux within the Earth," Proc. R. Soc. London, Ser. A 197, 433 (1949).

${ }^{2}$ W. V. R. Malkus, "Precession of the Earth as the cause of geomagnetism," Science 160, 259 (1968).

${ }^{3}$ R. R. Kerswell, "Upper bounds on the energy dissipation in turbulent precession,” J. Fluid Mech. 321, 335 (1996).

${ }^{4}$ J. P. Vanyo, P. Wilde, P. Cardin, and P. Olson, "Experiments on precessing flows in the Earth's liquid core," Geophys. J. Int. 121, 136 (1995).

${ }^{5}$ J. Noir, D. Jault, and P. Cardin, "Numerical study of the motions within a slowly precessing sphere at low Ekman number," J. Fluid Mech. 437, 283 (2001).

${ }^{6}$ J. Noir, P. Cardin, D. Jault, and J. P. Masson, "Experimental evidence of nonlinear resonance effects between retrograde precession and the tilt-over mode within a spheroid," Geophys. J. Int. 154, 407 (2003).

${ }^{7}$ S. Goto, N. Ishii, S. Kida, and M. Nishioka, "Turbulence generator using a precessing sphere," Phys. Fluids 19, 061705 (2007).

${ }^{8}$ A. Tilgner, "Precession-driven dynamos," Phys. Fluids 17, 034104 (2005).

${ }^{9}$ A. Tilgner, "Kinematic dynamos with precession-driven flow in a sphere," Geophys. Astrophys. Fluid Dyn. 101, 1 (2007).

${ }^{10}$ C. C. Wu and P. H. Roberts, "On a dynamo driven by topographic precession," Geophys. Astrophys. Fluid Dyn. 103, 467 (2009).

${ }^{11} \mathrm{~K}$. Stewartson and P. H. Roberts, "On the motion of a liquid in a spheroidal cavity of a precessing rigid body," J. Fluid Mech. 17, 1 (1963).

${ }^{12}$ P. H. Roberts and K. Stewartson, "On the motion of a liquid in a spheroidal cavity of a precessing rigid body. II," Math. Proc. Cambridge Philos. Soc. 61, 279 (1965)

${ }^{13}$ F. H. Busse, "Steady fluid flow in a precessing spheroidal shell," J. Fluid Mech. 33, 739 (1968). 
${ }^{14}$ J. P. Vanyo and P. W. Likins, "Rigid-body approximation to turbulent motion in a liquid-filled, precessing, spherical cavity," ASME J. Appl. Mech. 39, 19 (1972).

${ }^{15}$ R. Hollerbach and R. R. Kerswell, "Oscillatory internal shear layers in rotating and precessing flows," J. Fluid Mech. 298, 327 (1995).

${ }^{16}$ A. Tilgner, "Magnetohydrodynamic flow in precessing spherical shells," J. Fluid Mech. 379, 303 (1999).

${ }^{17}$ A. Tilgner and F. H. Busse, "Fluid flows in precessing spherical shells," J. Fluid Mech. 426, 387 (2001).

${ }^{18}$ A. Tilgner, in Core Dynamics, Rotational Dynamics of the Core Vol. 8, edited by G. Schubert (Elsevier, Amsterdam, 2007), pp. 207-243.

${ }^{19}$ H. P. Greenspan, The Theory of Rotating Fluids (Cambridge University Press, Cambridge, 1968).

${ }^{20} \mathrm{~K}$. Aldridge, "Inertial waves and the Earth's outer core," Geophys. J. R. Astron. Soc. 42, 337 (1975)
${ }^{21}$ K. Zhang, P. Earnshaw, X. Liao, and F. H. Busse, "On inertial waves in a rotating fluid sphere," J. Fluid Mech. 437, 103 (2001).

${ }^{22} \mathrm{~K}$. Zhang and X. Liao, "A new asymptotic method for the analysis of convection in a rotating sphere," J. Fluid Mech. 518, 319 (2004).

${ }^{23} \mathrm{~K}$. Zhang, "On coupling between the Poincaré equation and the heat equation: nonslip boundary condition,” J. Fluid Mech. 284, 239 (1995).

${ }^{24} \mathrm{~K}$. Chan, K. Zhang, L. Li, and X. Liao, "A new generation of convectiondriven spherical dynamos using EBE finite element method," Phys. Earth Planet. Inter. 163, 251 (2007).

${ }^{25}$ S. Lorenzani and A. Tilgner, "Fluid instabilities in precessing spheroidal cavities," J. Fluid Mech. 447, 111 (2001).

${ }^{26} \mathrm{~K}$. Zhang, X. Liao, and F. H. Busse, "Asymptotic solutions of convection in rapidly rotating nonslip spheres," J. Fluid Mech. 578, 371 (2007). 